\section{Treatment of anemic CKD patients improves outcomes and reduces health-care costs}

In a large retrospective claims analysis, Maddux et al. have shown that the use of erythropoiesisstimulating agents (ESAs) in anemic patients with CKD who are not receiving dialysis resulted in lower inpatient mortality, delayed initiation of dialysis and a mean total saving of US $\$ 411$ per patient per month.

The researchers reviewed claims data for the period 2000-2004 from a large US health plan database and identified 26,244 patients with CKD, $31.2 \%$ of whom had anemia. Among the patients with anemia, $14.6 \%(n=1,197)$ received ESAs. In comparison with anemic patients who did not receive ESAs, significantly fewer ESAtreated patients died during the study period $(P=0.0038)$ and the mean time to dialysis initiation was markedly prolonged (294 days vs 443 days; $P<0.0001)$. However, a greater proportion of ESA-treated patients experienced cardiovascular events and began dialysis.

Despite the increased ambulatory and pharmaceutical costs associated with office administration of ESAs, total monthly healthcare costs were $19 \%$ lower for ESA-treated patients than for untreated anemic patients $(P=0.0061)$. This cost saving is attributable to the considerably fewer inpatient and emergency-department visits made by ESA-treated patients. The authors conclude that the low rate of treatment for anemia in CKD should be addressed.

Original article Maddux FW et al. (2007) Effect of erythropoiesis-stimulating agents on healthcare utilization, costs, and outcomes in chronic kidney disease. Ann Pharmacother 41: 1761-1769

\section{Intravenous C.E.R.A. is as effective as standard epoetin in patients undergoing dialysis}

Two large randomized studies recently reported that intravenous methoxy polyethylene glycolepoetin beta (C.E.R.A; given every 2 or 4 weeks) is as safe and effective as conventional epoetin (given 1-3 times per week) for control of hemoglobin levels in patients on dialysis.

One trial randomized 223 hemodialysis patients to intravenous C.E.R.A. every 2 weeks, 224 patients to intravenous C.E.R.A. every 4 weeks, and 226 patients to standard treatment with epoetin alfa or beta 1-3 times per week, for approximately 1 year. All patients had received epoetin alfa or beta for 4 weeks before switching to C.E.R.A. or continuing on epoetin. Mean changes from baseline hemoglobin concentration were no different between those treated with standard epoetin therapy and those receiving intravenous C.E.R.A. either every 2 or 4 weeks. The adverse event profile was also similar within the groups.

The other study randomized dialysis patients who were naive to erythropoietinstimulating agents either to 24 weeks of intravenous C.E.R.A. administered every 2 weeks $(n=135)$ or to epoetin alfa or beta three times weekly $(n=46)$. Rates of hemoglobin response (defined as an increase in hemoglobin level of $\geq 10 \mathrm{~g} / \mathrm{l}$ and hemoglobin level $\geq 110 \mathrm{~g} / \mathrm{l}$ ) were similar in the C.E.R.A. and epoetin groups (93.3\% vs $91.3 \%$ ). Peak mean hemoglobin concentrations and changes in hemoglobin concentrations from baseline to the end of the treatment period were also comparable among groups. Both therapies were generally well tolerated.

\section{Original articles Levin NW et al. (2007) Intravenous methoxy polyethylene glycol-epoetin beta for haemoglobin control in patients with chronic kidney disease who are on dialysis: a randomised non-inferiority trial (MAXIMA). Lancet 370: 1415-1421 \\ Klinger M et al. (2007) Efficacy of intravenous methoxy polyethylene glycol-epoetin beta administered every 2 weeks compared with epoetin administered 3 times weekly in patients treated by hemodialysis or peritoneal dialysis: a randomized trial. Am J Kidney Dis 50: 989-1000 \\ Nicotinic acid controls serum phosphate and raises HDL levels in dialysis patients}

Many of the strategies that are employed routinely to manage serum phosphate levels in patients with end-stage renal disease have adverse effects. Müller et al. investigated in dialysis patients the therapeutic value of slowrelease nicotinic acid (also known as niacin and vitamin $B_{3}$ ), a dietary component that inhibits intestinal phosphate reabsorption and raises levels of HDL cholesterol. This latter effect might help to reduce cardiovascular risk.

The trial involved 20 dialysis patients who had been receiving a calcium-containing 